\title{
Distributions and the Global Operator of Slice Monogenic Functions
}

\author{
Fabrizio Colombo • Franciscus Sommen
}

Received: 16 June 2013 / Accepted: 16 July 2013 / Published online: 30 July 2013

\begin{abstract}
In this paper we consider functions $f$ defined on an open set $U$ of the Euclidean space $\mathbb{R}^{n+1}$ and with values in the Clifford Algebra $\mathbb{R}_{n}$. Slice monogenic functions $f: U \subseteq \mathbb{R}^{n+1} \rightarrow \mathbb{R}_{n}$ belong to the kernel of the global differential operator with non constant coefficients given by $\mathcal{G}=|\underline{x}|^{2} \frac{\partial}{\partial x_{0}}+\underline{x} \sum_{j=1}^{n} x_{j} \frac{\partial}{\partial x_{j}}$. Since the operator $\mathcal{G}$ is not elliptic and there is a degeneracy in $\underline{x}=0$, its kernel contains also less smooth functions that have to be interpreted as distributions. We study the distributional solutions of the differential equation $\mathcal{G} F\left(x_{0}, \underline{x}\right)=G\left(x_{0}, \underline{x}\right)$ and some of its variations. In particular, we focus our attention on the solutions of the differential equation $\left(\underline{x} \frac{\partial}{\partial x_{0}}-E\right) F\left(x_{0}, \underline{x}\right)=G\left(x_{0}, x\right)$, where $E=\sum_{j=1}^{n} x_{j} \frac{\partial}{\partial x_{j}}$ is the Euler operator, from which we deduce properties of the solutions of the equation $\mathcal{G} F\left(x_{0}, \underline{x}\right)=G\left(x_{0}, \underline{x}\right)$.
\end{abstract}

\author{
F. Colombo (ه) \\ Dipartimento di Matematica, Politecnico di Milano, Via Bonardi, 9, 20133 Milan, Italy \\ e-mail: fabrizio.colombo@polimi.it \\ F. Sommen \\ Clifford Research Group Faculty of Sciences, Ghent University, Galglaan 2, 9000 Gent, Belgium \\ e-mail: Frank.Sommen@UGent.be
}




\section{Introduction}

The global operator $\mathcal{G}$ associated with slice monogenic functions has been recently introduced in [5]. This operator contains in its kernel the set of slice monogenic functions and, when $n=3$ and $x$ is the imaginary part of a quaternion, the set of slice regular functions which is the quaternionic version of this theory. Both classes of functions are called slice hyperholomorphic functions. There are several applications of slice hyperholomorphic functions in functional analysis and in operator theory, in particular to Schur analysis [1-3]. Moreover with these functions we can define the so called S-functional calculus, which is the non commutative version of the RieszDunford calculus for $n$-tuples of non necessarily commuting operators, see $[6,9,10]$. In this paper, we begin the study of the distributional solutions the equation $\mathcal{G} F=G$, where $F$ and $G$ are Clifford algebra valued functions. The problem is non trivial since the operator $\mathcal{G}$ has non constant coefficients and is degenerate. In [12], it has been recently introduced a system of differential equations which generalize the system $\mathcal{G} F=0$ to the case of slice regular functions with values in an alternative real algebra.

Let us denote by $\mathbb{R}_{n}$ the real Clifford algebra over $n$ imaginary units $e_{1}, \ldots, e_{n}$ satisfying the relations $e_{i} e_{j}+e_{j} e_{i}=-2 \delta_{i j}$. An element in the Clifford algebra $\mathbb{R}_{n}$ will be denoted by $\sum_{A} e_{A} x_{A}$ where $A=i_{1} \ldots i_{r}, i_{\ell} \in\{1,2, \ldots, n\}, i_{1}<\ldots<i_{r}$ is a multi-index, $e_{A}=e_{i_{1}} e_{i_{2}} \ldots e_{i_{r}}$ and $e_{\emptyset}=1, x_{A} \in \mathbb{R}$. Some specific elements in $\mathbb{R}_{n}$ can be identified with vectors in the Euclidean space $\mathbb{R}^{n}$ : an element $\left(x_{1}, x_{2}, \ldots, x_{n}\right) \in$ $\mathbb{R}^{n}$ can be identified with a so called 1 -vector in the Clifford algebra through the map $\left(x_{1}, x_{2}, \ldots, x_{n}\right) \mapsto \underline{x}=x_{1} e_{1}+\cdots+x_{n} e_{n}$.

The element $\left(x_{0}, x_{1}, \ldots, x_{n}\right) \in \mathbb{R}^{n+1}$ will be identified with $x=x_{0}+\underline{x}=x_{0}+$ $\sum_{j=1}^{n} x_{j} e_{j}$, and is called paravector. The norm of $x \in \mathbb{R}^{n+1}$ is defined as $|x|^{2}=$ $x_{0}^{2}+x_{1}^{2}+\cdots+x_{n}^{2}$. The real part $x_{0}$ of $x$ will be also written as $\operatorname{Re}(x)$. A function $f: U \subseteq \mathbb{R}^{n+1} \rightarrow \mathbb{R}_{n}$ is treated as a function $f(x)$, or more explicitly $f\left(x_{0}, \underline{x}\right)$, of the paravector $x$. The symbol $\mathbb{S}$ will denote the sphere of unit 1 -vectors in $\mathbb{R}^{n}$, i.e.

$$
\mathbb{S}=\left\{\underline{x}=e_{1} x_{1}+\cdots+e_{n} x_{n}: x_{1}^{2}+\cdots+x_{n}^{2}=1\right\} .
$$

Note that $\mathbb{S}$ is an $(n-1)$-dimensional sphere in $\mathbb{R}^{n+1}$ lying on the hyperplane $x_{0}=0$ that can be identified with the Euclidean space $\mathbb{R}^{n}$.

The set of slice monogenic functions is defined as follows, see [8]:

Definition 1.1 Let $U \subseteq \mathbb{R}^{n+1}$ be an open set and let $f: U \rightarrow \mathbb{R}_{n}$ be a real differentiable function. Let $I \in \mathbb{S}$ and let $f_{I}$ be the restriction of $f$ to the complex plane $\mathbb{C}_{I}$, where an element in $\mathbb{C}_{I}$ is denoted by $u+I v, u, v \in \mathbb{R}$. We say that $f$ is a (left) slice monogenic function, or s-monogenic function, if for every $I \in \mathbb{S}$, we have

$$
\frac{1}{2}\left(\frac{\partial}{\partial u}+I \frac{\partial}{\partial v}\right) f_{I}(u+I v)=0
$$

We denote by $\mathcal{S M}(U)$ the set of s-monogenic functions on $U$. 
We note that in the literature there is another notion of monogenic functions which is already defined by a global operator, with constant coefficients, called Dirac operator, see $[4,7,11]$.

In [5] it has been introduced the following operator

$$
\mathcal{G}=|\underline{x}|^{2} \frac{\partial}{\partial x_{0}}+\underline{x} \sum_{j=1}^{n} x_{j} \frac{\partial}{\partial x_{j}}
$$

which acts on functions $f: U \rightarrow \mathbb{R}_{n}$, which have a suitable regularity, where $U \subseteq \mathbb{R}^{n+1}$ is an open set. It has been shown that the set of slice monogenic functions $\mathcal{S} \mathcal{M}(U)$ is contained in the kernel of $\mathcal{G}$. But since the operator is non elliptic, we have also solutions that are not necessarily slice monogenic functions but can be much less smooth. Moreover there is a degeneracy for $\underline{x}=0$. If we denote by $E$ the Euler operator

$$
E=\sum_{j=1}^{n} x_{j} \frac{\partial}{\partial x_{j}}
$$

from (1) we deduce that we can also consider, for any fixed $n \in \mathbb{N}$, an operator which is less degenerate, that is

$$
\underline{x} \frac{\partial}{\partial x_{0}}-E,
$$

but also operators that are more degenerate like

$$
\underline{x}^{p}\left(x \frac{\partial}{\partial x_{0}}-E\right) \text { for } p \in \mathbb{N}
$$

which coincide, for $p=1$, with the operator $\mathcal{G}$.

The case $n=1$ is studied in Sect. 2, while in Sect. 3 and in Sect. 4 we show some results in the higher dimensional case.

\section{The 1-Dimensional Case}

We recall two main facts on the theory of distributions that are very well known but will be important for our formulation of the problem. For the theory of distributions we refer the reader to [14, p. 52].

Theorem 2.1 Let $\Omega \subseteq \mathbb{R}$ be an open set. A linear functional $f$ on $\mathcal{D}(\Omega)$ is a distribution if and only if for every open set $\Omega^{\prime} \subset \Omega$, with $\bar{\Omega}^{\prime} \subset \Omega$ and $\bar{\Omega}^{\prime}$ compact, there exist two numbers $K=K\left(\Omega^{\prime}\right)$ and $m=m\left(\Omega^{\prime}\right)$ such that

$$
|\langle f, \varphi\rangle| \leq K\|\varphi\|_{C^{m}\left(\overline{\Omega^{\prime}}\right)} .
$$


From the above theorem naturally arises the definition of the order of a distribution.

Definition 2.2 Suppose that $f \in \mathcal{D}^{\prime}(\Omega)$, where $\Omega \subseteq \mathbb{R}$, and suppose that we can choose the integer number $m$ independently of $\Omega^{\prime}$ such that (4) holds. We will say that $f$ is of finite order and the minimum integer $m$ such that (4) holds is called the order of the distribution $f$.

Theorem 2.3 (Distributions with support in a point) Let $f \in \mathcal{D}^{\prime}(\Omega)$, where $\Omega \subseteq \mathbb{R}$, and suppose that $\operatorname{supp} f$ is the point 0 . Then $f$ is uniquely represented by the formula

$$
f(x)=\sum_{k=0}^{m} c_{k} D^{k} \delta(x)
$$

where $m$ is the order of $f, c_{k}$ are constants, and $D$ denotes the derivative with respect to $x$.

Finally we recall that the equation $x^{N} f(x)=0$ in $\mathcal{D}^{\prime}(\mathbb{R})$ has the solutions

$$
f(x)=\sum_{k=0}^{N-1} c_{k} D^{k} \delta(x)
$$

where $c_{k}$ are arbitrary constants.

For the case $n=1$ we can study explicitly the kernel of the operator $\left(\underline{x} \partial_{x_{0}}-E\right)$. With the positions $x_{0}=x, x_{1}=y$ and $e_{1}=i$ we have

$$
y\left(\partial_{x}+i \partial_{y}\right) f(x, y)=0 .
$$

Theorem 2.4 In $\mathcal{D}^{\prime}\left(\mathbb{R}^{2}\right)$ the general solution of (5) is locally given by

$$
f(x, y)=h(x, y)+\mathcal{E} * c(x) \delta(y)
$$

where $h$ is any holomorphic function and $c(x)$ is an arbitrary distribution, with compact support, in $x$ and $\mathcal{E}$ is the fundamental solution of the Cauchy-Riemann operator. Proof Solving the division problem for $y\left(\partial_{x}+i \partial_{y}\right) f(x, y)=0$ we get

$$
\left(\partial_{x}+i \partial_{y}\right) f(x, y)=c(x) \delta(y)
$$

where locally, e.g. for $|x+i y|<R$ for a given $R>0$, we may assume the support of $c(x) \delta(y)$ to be compact.

As the solutions of the kernel of $\left(\partial_{x}+i \partial_{y}\right)$ are the holomorphic functions, we get the statement by standard arguments. In fact, let $\mathcal{E}$ be the fundamental solution of the Cauchy-Riemann operator, $\left(\partial_{x}+i \partial_{y}\right) \mathcal{E}(z)=\delta_{0}$ then the solution of $\left(\partial_{x}+\right.$ $\left.i \partial_{y}\right) F(x, y)=c(x) \delta(y)$ is given by

$$
F(x, y)=\mathcal{E} * c(x) \delta(y) .
$$


The convolution is well defined since $c(x) \delta(y)$ has compact support. So $F$ plus the solutions in the kernel of the Cauchy-Riemann operator gives the general solution.

Remark 2.5 Thanks to the above results, with the same technique, we can study the homogeneous equation:

$$
y^{p}\left(\partial_{x}+i \partial_{y}\right) f(x, y)=0, \quad p=2,3, \ldots
$$

In fact, it can be written as

$$
\left(\partial_{x}+i \partial_{y}\right) f(x, y)=\sum_{k=0}^{p-1} c_{k}(x) D^{k} \delta(y)
$$

since distributions with compact support on the real line, are represented by

$$
\sum_{k=0}^{q} c_{k}(x) D^{k} \delta(y)
$$

for suitable $q$ and where $c_{k}(x)$ have compact support as well.

Remark 2.6 For $n=1$ the paravector variable $x_{0}+\underline{x}$ becomes the complex variable $z$, and for $m$ complex variables, denoted by $z_{j}=x_{j}+i y_{j}, x_{j}, y_{j} \in \mathbb{R}$, we can consider a function $f=f\left(z_{1}, z_{2}, \ldots z_{m}\right)$ defined in an open set in $\mathbb{R}^{2 m}$. So for the several variables case the system to be considered becomes

$$
y_{j}\left(\partial_{x_{j}}+i \partial_{y_{j}}\right) f\left(z_{1}, z_{2}, \ldots, z_{m}\right)=0, \quad j=1, \ldots, m \text { and } m \in \mathbb{N} \text {. }
$$

It can be also studied by solving the division problem in the sense of distributions in several variables.

\section{The Higher Dimensional Case}

We now consider the nonconstant coefficients differential operator

$$
\underline{x} \partial_{x_{0}}-E
$$

where $E$ is the Euler operator for $n \geq 2$. In this case things are more complicated as it can be seen explicitly by writing the equation $\left(\underline{x} \partial_{x_{0}}-E\right) f=0$ for $n=2$ in components. By setting $f=f_{0}+f_{1} e_{1}+f_{2} e_{2}+f_{3} e_{1} e_{2}$ where $f_{j}: \mathbb{R}^{2} \rightarrow \mathbb{R}, j=$ $0, \ldots, 3$ we have the system

$$
\left\{\begin{array}{l}
x_{1}\left(\partial_{0} f_{1}+\partial_{1} f_{0}\right)+x_{2}\left(\partial_{0} f_{2}+\partial_{2} f_{0}\right)=0 \\
x_{1}\left(\partial_{0} f_{0}-\partial_{1} f_{1}\right)+x_{2}\left(\partial_{0} f_{3}-\partial_{2} f_{1}\right)=0 \\
x_{1}\left(\partial_{0} f_{3}+\partial_{1} f_{1}\right)-x_{2}\left(\partial_{0} f_{0}-\partial_{2} f_{2}\right)=0 \\
x_{1}\left(\partial_{0} f_{2}-\partial_{1} f_{3}\right)-x_{2}\left(\partial_{0} f_{1}+\partial_{2} f_{3}\right)=0
\end{array}\right.
$$


where we have set $\partial_{j}=\partial_{x_{j}}, j=0, \ldots, 3$. Thus we cannot use the division strategy as in the 1-dimensional case.

Let us recall some properties of the Euler operator

$$
E=\sum_{j=1}^{n} x_{j} \frac{\partial}{\partial x_{j}}
$$

in the setting of distributions. These results are in [11, p. 351].

Lemma 3.1 Let $\partial_{\underline{x}}=\sum_{j=1}^{n} e_{j} \partial_{x_{j}}$ be the Dirac operator in dimension $n \in \mathbb{N}$ and let $\ell \in \mathbb{N}$. Then

$$
E\left[\partial_{\underline{x}}^{\ell} \delta(\underline{x})\right]=-(n+\ell) \partial_{\underline{x}}^{\ell} \delta(\underline{x})
$$

where $\delta(\underline{x})$ denotes the delta distribution.

Proof It follows from the fact that $\delta(\underline{x})$ is homogeneous of degree $-n$ and the fact that the Euler operator measures the degree of homogeneity.

In the Clifford algebras setting the structure theorem of distributions supported at the origin is given by:

Theorem 3.2 Let $F \in \mathcal{D}^{\prime}\left(\mathbb{R}^{n+1}\right)$ and suppose that $\operatorname{supp} F$ is compact and contained in the real axis identified with $\left\{x \in \mathbb{R}^{n+1}: \underline{x}=0\right\}$. Then $F$ is uniquely represented by the formula

$$
F\left(x_{0}, \underline{x}\right)=\sum_{|\alpha| \leq N} A_{\alpha}\left(x_{0}\right) \partial_{\underline{x}}^{\alpha} \delta(\underline{x}),
$$

where $N$ is the order of $F$ and $A_{\alpha}$ are distributions, with compact support, depending on $x_{0}$.

We have the following result:

Theorem 3.3 Let $G$ be a given distribution in $\mathcal{D}^{\prime}\left(\mathbb{R}^{n+1}\right)$ with compact support in the real axis identified with $\left\{x \in \mathbb{R}^{n+1}: \underline{x}=0\right\}$. Suppose that the solution $F\left(x_{0}, \underline{x}\right)$ to the equation

$$
\left(\underline{x} \partial_{x_{0}}-E\right) F\left(x_{0}, \underline{x}\right)=G\left(x_{0}, \underline{x}\right),
$$

has compact support in $\mathbb{R}$. Then $F$ is unique.

Proof Since $G$ is with compact support for the previous theorem we can write it as

$$
G\left(x_{0}, \underline{x}\right)=\sum_{s=0}^{\ell} G_{-n-s}\left(x_{0}, \underline{x}\right),
$$


where

$$
G_{-n-s}\left(x_{0}, \underline{x}\right)=\sum_{|\alpha|=s} G_{\alpha}\left(x_{0}\right) \partial_{\underline{x}}^{\alpha} \delta(\underline{x}) .
$$

Here each $G_{-n-s}\left(x_{0}, \underline{x}\right)$ is homogeneous of degree $-(n+s)$, in the $\underline{x}$ variable. This notation will be useful in the sequel. Since also $F\left(x_{0}, \underline{x}\right)$ has compact support we can write

$$
F\left(x_{0}, \underline{x}\right)=\sum_{s=0}^{\ell} F_{-n-s}\left(x_{0}, \underline{x}\right)
$$

and each $F_{-n-s}\left(x_{0}, \underline{x}\right)$ is homogeneous of degree $-(n+s)$ in the $\underline{x}$ variable. Thanks to Lemma 3.1 we have

$$
\begin{gathered}
E F\left(x_{0}, \underline{x}\right)=-n F_{-n}\left(x_{0}, \underline{x}\right)-(n+1) F_{-n-1}\left(x_{0}, \underline{x}\right)-\cdots \\
\cdots-(n+\ell-1) F_{-n-s+1}\left(x_{0}, \underline{x}\right)-(n+\ell) F_{-n-\ell}\left(x_{0}, \underline{x}\right) .
\end{gathered}
$$

Since

$$
\partial_{x_{0}} \underline{x} F\left(x_{0}, \underline{x}\right)=\underline{x} \partial_{x_{0}} F\left(x_{0}, \underline{x}\right)
$$

we have that $\partial_{x_{0}} \underline{x} F\left(x_{0}, \underline{x}\right)$ can be decomposed as the sum of $\partial_{x_{0}} \underline{x} F_{S}\left(x_{0}, \underline{x}\right)$ each term being homogeneous of degree $-(m+s-1)$, in the $\underline{x}$ variable. By substituting in (10) we obtain

$$
\partial_{x_{0}} \underline{x} \sum_{s=0}^{\ell} F_{-n-s}\left(x_{0}, \underline{x}\right)-\sum_{s=0}^{\ell} E F_{-n-s}\left(x_{0}, \underline{x}\right)=\sum_{s=0}^{\ell} G_{-n-s}\left(x_{0}, \underline{x}\right) .
$$

By writing explicitly the terms in this equality we have

$$
\begin{aligned}
& \partial_{x_{0}} \underline{x} F_{-n}\left(x_{0}, \underline{x}\right)+\partial_{x_{0}} \underline{x} F_{-n-1}\left(x_{0}, \underline{x}\right) \\
& \quad+\cdots+\partial_{x_{0}} \underline{x} F_{-n-\ell+1}\left(x_{0}, \underline{x}\right)+\partial_{x_{0}} \underline{x} F_{-n-\ell}\left(x_{0}, \underline{x}\right) \\
& \quad-n F_{-n}\left(x_{0}, \underline{x}\right)-(n+1) F_{-n-1}\left(x_{0}, \underline{x}\right)-\cdots \\
& \quad \cdots-(n+\ell-1) F_{-n-s+1}\left(x_{0}, \underline{x}\right)-(n+\ell) F_{-n-\ell}\left(x_{0}, \underline{x}\right) \\
& \quad=G_{-n}\left(x_{0}, \underline{x}\right)+G_{-n-1}\left(x_{0}, \underline{x}\right)+\cdots+G_{-n-\ell+1}\left(x_{0}, \underline{x}\right)+G_{-n-\ell}\left(x_{0}, \underline{x}\right),
\end{aligned}
$$

and we can write the equalities for the terms with the same degree of homogeneity. In particular, for the degree $-n-\ell$, we have:

$$
-(n+\ell) F_{-n-\ell}\left(x_{0}, \underline{x}\right)=G_{-n-\ell}\left(x_{0}, \underline{x}\right)
$$


which gives

$$
F_{-n-\ell}\left(x_{0}, \underline{x}\right)=-\frac{1}{n+\ell} G_{-n-\ell}\left(x_{0}, \underline{x}\right) .
$$

Now observe that the term $\partial_{x_{0}} \underline{x} F_{-n-\ell}\left(x_{0}, \underline{x}\right)$ has degree of homogeneity $-n-\ell+1$. So we deduce the equation for the term of degree of homogeneity $-n-\ell+1$, that is

$$
\partial_{x_{0}} \underline{x} F_{-n-\ell}\left(x_{0}, \underline{x}\right)-(n+\ell-1) F_{-n-s+1}\left(x_{0}, \underline{x}\right)=G_{-n-\ell+1}\left(x_{0}, \underline{x}\right) .
$$

By replacing $F_{-n-\ell}\left(x_{0}, \underline{x}\right)=-\frac{1}{n+\ell} G_{-n-\ell}\left(x_{0}, \underline{x}\right)$ on the left hand side we obtain

$$
-\frac{1}{n+\ell} \partial_{x_{0}} \underline{x} G_{-n-\ell}\left(x_{0}, \underline{x}\right)-(n+\ell-1) F_{-n-s+1}\left(x_{0}, \underline{x}\right)=G_{-n-\ell+1}\left(x_{0}, \underline{x}\right)
$$

so we solve to get $F_{-n-s+1}\left(x_{0}, \underline{x}\right)$.

By iterating this procedure, in a finite number of steps, we determine all the terms $F_{-n-s}\left(x_{0}, \underline{x}\right)$, for $s \leq \ell$. Thus the uniqueness of the solution follows by construction.

Corollary 3.4 Let $p \in \mathbb{N}$ and suppose that in $\mathcal{D}^{\prime}\left(\mathbb{R}^{n+1}\right)$, the solution $F\left(x_{0}, \underline{x}\right)$ to the equation

$$
\underline{x}^{p}\left(\underline{x} \partial_{x_{0}}-E\right) F\left(x_{0}, \underline{x}\right)=0,
$$

has compact support in $\mathbb{R}$. Then $F$ is unique.

Proof Let $p \in \mathbb{N}$ then, by solving the division problem in the distributions $\mathcal{D}^{\prime}\left(\mathbb{R}^{n+1}\right)$, the equation

$$
\underline{x}^{p}\left(\underline{x} \partial_{x_{0}}-E\right) F\left(x_{0}, \underline{x}\right)=0,
$$

becomes equivalent to

$$
\left(\underline{x} \partial_{x_{0}}-E\right) F\left(x_{0}, \underline{x}\right)=\sum_{s=0}^{p-1} G_{-n-s}\left(x_{0}, \underline{x}\right)
$$

where

$$
G_{-n-s}\left(x_{0}, \underline{x}\right)=\sum_{|\alpha|=s} G_{\alpha}\left(x_{0}\right) \partial_{\underline{x}}^{\alpha} \delta(\underline{x})
$$

From Theorem 3.3 we get the statement.

Remark 3.5 The proof of Theorem 3.3 cannot be adapted to the case hyperfunctions. In fact suppose to take an hyperfunction supported at the origin. Then (see [13, p. 156]) 
its representation is a series of the derivatives of the delta of Dirac in the Clifford algebra case:

$$
\mathcal{G}\left(x_{0}, \underline{x}\right)=\sum_{s=0}^{\infty} \mathcal{G}_{-n-s}\left(x_{0}, \underline{x}\right),
$$

where

$$
\mathcal{G}_{-n-s}\left(x_{0}, \underline{x}\right)=\sum_{|\alpha|=s} \mathcal{G}_{\alpha}\left(x_{0}\right) \partial_{\underline{x}}^{\alpha} \delta(\underline{x}) .
$$

Thus, in this case, we do not have the minimum degree of homogeneity to start with. And so the procedure does not apply.

\section{The Noncompact Support Case}

Theorem 4.1 Let $\Omega$ be an open set in $\mathbb{R}^{n+1}$. Consider in $\mathcal{D}^{\prime}(\Omega)$ the differential equation

$$
\left(\underline{x} \partial_{x_{0}}-E\right) F\left(x_{0}, \underline{x}\right)=G\left(x_{0}, \underline{x}\right),
$$

and assume that the distribution $G$ is such that $\operatorname{supp}(G) \subseteq \Omega \cap\left\{x \in \mathbb{R}^{n+1} \mid \underline{x}=0\right\} \cong$ $\Omega \cap \mathbb{R}$. Then there exists a unique distribution $F$ with support in $\Omega \cap \mathbb{R}$ solving the Eq. (14).

Proof Let $\Omega \subset \mathbb{R}^{n+1}$ be an open set and assume that $\Omega^{\top} \subset \Omega$ where $\Omega^{\prime}$ is open and $\bar{\Omega}^{\prime}$ is compact. Then we may write like in the proof of Theorem 3.3

$$
\left.G\left(x_{0}, \underline{x}\right)\right|_{\Omega^{\prime}}=\sum_{s=0}^{\ell} G_{-n-s}\left(x_{0}, \underline{x}\right)
$$

where $\ell$ depends on $\Omega^{\prime}$ and $G_{-n-s}\left(x_{0}, \underline{x}\right)=\sum_{|\alpha|=s} G_{\alpha}\left(x_{0}\right) \partial_{\underline{x}}^{\alpha} \delta(\underline{x})$. Here each $G_{-n-s}\left(x_{0}, \underline{x}\right)$ is homogeneous of degree $-(n+s)$ in the $\underline{x}$ variable. If $F$ is a solution to the Eq. (14) supported by $\mathbb{R} \cap \Omega$, then

$$
\left.F\left(x_{0}, \underline{x}\right)\right|_{\Omega^{\prime}}=\sum_{s=0}^{\ell^{\prime}} F_{-n-s}\left(x_{0}, \underline{x}\right)
$$

for some $\ell^{\prime}$ that depends on $\Omega^{\prime}$ where each $F_{-n-s}\left(x_{0}, \underline{x}\right)$ is homogeneous of degree $-(n+s)$ in the $\underline{x}$ variable. For $\ell^{\prime}=\ell$ the functions $F_{-n-s}\left(x_{0}, \underline{x}\right)$ are uniquely determined by $G_{-n-s}\left(x_{0}, \underline{x}\right)$. Hence $\left.F\right|_{\Omega^{\prime}}$ exists and is unique for all $\Omega^{\prime}$. Now observe that for $\Omega^{\prime \prime} \subset \Omega^{\prime}$, where $\Omega^{\prime}$ and $\Omega^{\prime \prime}$ are open sets with $\bar{\Omega}^{\prime}$ and $\bar{\Omega}^{\prime \prime}$ compact sets, by uniqueness we have 


$$
\left.F\left(x_{0}, \underline{x}\right)\right|_{\Omega^{\prime \prime}}=\left.\left.F\left(x_{0}, \underline{x}\right)\right|_{\Omega^{\prime}}\right|_{\Omega^{\prime \prime}}
$$

So $F$ is well defined and unique on $\Omega$.

Theorem 4.2 Let $\Omega$ be an open set in $\mathbb{R}^{n+1}$. Suppose that there exists a solution $F$ to the equation

$$
\left(\underline{x} \partial_{x_{0}}-E\right) F\left(x_{0}, \underline{x}\right)=0, \quad x \in \Omega \backslash \mathbb{R}
$$

and assume that the solution $F$ extends to a distribution $\breve{F}$ defined on all $\Omega$. Then there exists a unique distributional extension $\tilde{F}$ of $F$ to all $\Omega$ satisfying

$$
\left(\underline{x} \partial_{x_{0}}-E\right) \tilde{F}\left(x_{0}, \underline{x}\right)=0 .
$$

Proof By assumption we have the existence of a distribution $\breve{F}$ extending $F$ to $\Omega$. Then, as

$$
\left(\underline{x} \partial_{x_{0}}-E\right) \breve{F}\left(x_{0}, \underline{x}\right)=0, \quad x \in \Omega \backslash \mathbb{R},
$$

the function $\left(x \partial_{x_{0}}-E\right) \breve{F}\left(x_{0}, \underline{x}\right)$ has support in $\Omega \cap \mathbb{R}$. Set $\left(\underline{x} \partial_{x_{0}}-E\right) \breve{F}\left(x_{0}, \underline{x}\right)=$ $G\left(x_{0}, \underline{x}\right)$ and consider now the equation

$$
\left(\underline{x} \partial_{x_{0}}-E\right) H\left(x_{0}, \underline{x}\right)=G\left(x_{0}, \underline{x}\right)
$$

on $\Omega \cap \mathbb{R}$. By Theorem 4.1 there exists a unique $H$ supported in $\mathbb{R}$ that solves the equation. By setting $\tilde{F}\left(x_{0}, \underline{x}\right)=\breve{F}\left(x_{0}, \underline{x}\right)-H\left(x_{0}, \underline{x}\right)$ we get the result.

Corollary 4.3 Let us consider in $\Omega \subseteq \mathbb{R}^{n+1}$ the differential equation associated to the operator (1):

$$
\left(|\underline{x}|^{2} \partial_{x_{0}}+\underline{x} \sum_{j=1}^{n} x_{j} \partial_{x_{j}}\right) F\left(x_{0}, \underline{x}\right)=0 .
$$

Then $F$ is a distributional solution in $\Omega$ if and only if $G$ defined by

$$
G\left(x_{0}, \underline{x}\right)=\left(\underline{x} \partial_{x_{0}}-E\right) F\left(x_{0}, \underline{x}\right)
$$

is a distributional solution of $\underline{x} G\left(x_{0}, \underline{x}\right)=0$.

Proof It is a consequence of the previous results.

Remark 4.4 The above implies that supp $G \subseteq \Omega \bigcap \mathbb{R}$. Now

$$
\left.\left(\underline{x} \partial_{x_{0}}-E\right) F\left(x_{0}, \underline{x}\right)\right|_{\Omega \backslash \mathbb{R}}=0
$$


and $\left.F\left(x_{0}, x\right)\right|_{\Omega \backslash \mathbb{R}}$ extends to $F$. So Theorem 4.2 implies the existence and uniqueness of $\tilde{F} \in \mathcal{D}^{\prime}(\Omega)$ with $\left(\underline{x} \partial_{x_{0}}-E\right) \tilde{F}\left(x_{0}, \underline{x}\right)=0$ in $\mathcal{D}^{\prime}(\Omega)$. So $H\left(x_{0}, \underline{x}\right)=F\left(x_{0}, \underline{x}\right)-$ $\tilde{F}\left(x_{0}, \underline{x}\right)$ satisfies

$$
\left(\underline{x} \partial_{x_{0}}-E\right) H\left(x_{0}, \underline{x}\right)=G\left(x_{0}, \underline{x}\right)
$$

and $\operatorname{supp} H \subset \Omega \bigcap \mathbb{R}$. So we conclude that

$$
F\left(x_{0}, \underline{x}\right)=\tilde{F}\left(x_{0}, \underline{x}\right)+H\left(x_{0}, \underline{x}\right)
$$

with

$$
\left(\underline{x} \partial_{x_{0}}-E\right) \tilde{F}\left(x_{0}, \underline{x}\right) \equiv 0
$$

and $\operatorname{supp} H \subset \Omega \bigcap \mathbb{R}$.

Example 4.5 If we consider $\left(x_{0}+x\right)^{-\ell}, \ell \leq n$ then it is locally integrable on $\mathbb{R}^{n+1}$ and so it is a distribution. By the Theorem $\overline{3} .3$, it solves the homogeneous equation associated to (14) identically.

Remark 4.6 For $\ell>n$, these functions $\left(x_{0}+\underline{x}\right)^{-\ell}$, are not regular distributions, but there exists the possibility to suitably "correct them " in order to get a distribution still solution to the differential equation. For example in the case $\ell=n+1$ we have that the distribution solution to the equation is of the form $\left(x_{0}+\underline{x}\right)^{-\ell}+c \delta$ where $c$ has to be chosen in order to have a solution of homogeneous equation associated to (14).

Remark 4.7 It is then natural to ask when is it true that a function of the form

$$
F\left(x_{0}, \underline{x}\right)=P\left(x_{0}, \underline{x}\right) /\left(x_{0}^{2}+|\underline{x}|^{2}\right)^{\ell}
$$

solves the homogeneous differential equation $\left(\underline{x} \partial_{x_{0}}-E\right) F\left(x_{0}, x\right)$, where $P\left(x_{0}, \underline{x}\right)$ is a suitable polynomial.

\section{References}

1. Alpay, D., Colombo, F., Sabadini, I.: Schur functions and their realizations in the slice hyperholomorphic setting. Integr. Equ. Oper. Theory 72, 253-289 (2012)

2. Alpay, D., Colombo, F., Sabadini, I.: Pontryagin-de Branges-Rovnyak spaces of slice hyperholomorphic functions. J. Anal. Math. (2013, to appear)

3. Alpay, D., Colombo, F., Sabadini, I.: Krein-Langer factorization and related topics in the slice hyperholomorphic setting. J. Geom. Anal. (2012). doi:10.1007/s12220-012-9358-5

4. Brackx, F., Delanghe, R., Sommen, F.: Clifford analysis. Pitman Res. Notes Math. 76 (1982)

5. Colombo, F., Gonzalez-Cervantes, J.O., Sabadini, I.: A nonconstant coefficients differential operator associated to slice monogenic functions. Trans. Am. Math. Soc. 365, 303-318 (2013)

6. Colombo, F., Sabadini, I.: The Cauchy formula with $s$-monogenic kernel and a functional calculus for noncommuting operators. J. Math. Anal. Appl. 373, 655-679 (2011)

7. Colombo, F., Sabadini, I., Sommen, F., Struppa, D.C.: Analysis of Dirac Systems and Computational Algebra, Progress in Mathematical Physics, vol. 39. Birkhäuser, Boston (2004)

8. Colombo, F., Sabadini, I., Struppa, D.C.: Slice monogenic functions. Isr. J. Math. 171, 385-403 (2009) 
9. Colombo, F., Sabadini, I., Struppa, D.C.: A new functional calculus for noncommuting operators. J. Funct. Anal. 254, 2255-2274 (2008)

10. Colombo, F., Sabadini, I., Struppa, D.C.: Noncommutative Functional Calculus. Theory and Applications of Slice Hyperholomorphic Functions, Progress in Mathematics, vol. 289, Birkhäuser, Basel (2011)

11. Delanghe, R., Sommen, F., Souček, V.: Clifford Algebra and Spinor-valued Functions, Mathematics and Its Applications, vol. 53. Kluwer Academic Publishers, Dordrecht (1992)

12. Ghiloni, R., Perotti, A.: Global differential equations for slice regular functions (2013, preprint)

13. Kaneko, A.: Introduction to Hyperfunctions. Kluwer Academic Publishers Group, Dordrecht (1988)

14. Vladimirov, V.: Distributions en physique mathématique. Translated from the Russian, "Mir", Moscow (1979) 